\title{
Extra-abdominal desmoid tumor: A case report
}

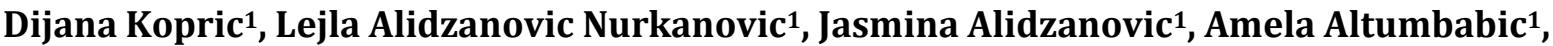 Lejla Arnautalic ${ }^{2}$, Ermina Iljazovic ${ }^{3}$}

\author{
${ }^{1}$ Department of Medical Oncology, University Clinical Center Tuzla, Tuzla, Bosnia and Herzegovina \\ 2 Department of Radiology, University Clinical Center Tuzla, Tuzla, Bosnia and Herzegovina \\ ${ }^{3}$ Department of Pathology, University Clinical Center Tuzla, Tuzla, Bosnia and Herzegovina
}

Received February 02, 2016; Revised July 09, 2016; Accepted July 12, 2016; Published Online July 16, 2016

\section{Case Report}

\begin{abstract}
Desmoid tumor represents a rare monoclonal, fibroblastic proliferation characterized by a variable and often unpredictable clinical course. Although histologically benign, desmoids are locally invasive and associated with a high local recurrence rate, but lack of metastatic potential. Many issues regarding the optimal treatment of patients with desmoids remain controversial. Surgical resection and radiotherapy are standard treatment options for these patients. Due to heterogeneity of the biological behavior of desmoids, including long periods of stable disease or even spontaneous regression, treatment needs to be individualized to optimize local tumor control and preserve patient's quality of life. Therapeutic approaches to the treatment of recurrent or unresectable desmoid tumors comprise anti-hormonal therapy, non-steroidal anti-inflammatory drugs, classic chemotherapy regimens and tyrosine kinase inhibitor, with highly variable results. It has not yet been possible to establish an optimal therapy protocol for this disease. In this case report we present our experience with the treatment of recurrent extra-abdominal desmoid tumor.
\end{abstract}

Keywords: Aggressive fibromatosis, Extra-abdominal, Desmoid tumor, Irradiation, Imatinib

\section{Introduction}

Desmoid tumors (DT), also known as aggressive fibromatosis or musculoaponeurotic fibromatosis, are fibroblastic tumors of intermediate malignant potential. They are characterized by slow growth, tendency for local recurrence and absence of metastatic potential. The term "desmoid", coined by Muller in 1838, is derived from the Greek word "desmos", meaning tendon-like. DTs are extremely rare and account for less than $0.03 \%$ of all tumors ${ }^{1}$. Surgical resection and radiotherapy are standard treatment options for these patients $^{2-4}$. Recurrence rate is high during DT management. Systemic treatment with Imatinib for unresectable or recurrent DTs could be acceptable therapeutic option ${ }^{5}$.

This report depicts a case of extra-abdominal DT of the gluteal region in a 43-year-old female who has reported to the oncologist for further treatment after a surgical resection and a year after subsequent disease recurrence. The goal of this case report is to provide better insight in treatment options of this rare disease and to emphasize the importance of multidisciplinary approach in the treatment of such patients.

\section{Case Presentation}

A 43-year-old female underwent surgical resection of the tumor in the right gluteal region at the Orthopedic Clinic in September 2010. Tumor was previously diagnosed by MRI scan. Upon surgical examination of the pelvic region, the tumor was found to be located between the right medial and large gluteal muscles, anterior and caudal to greater trochanter. Differential diagnosis suggested aggressive fibromatosis. Total extirpation of tumor was performed, and upon receiving a pathology report, patient was not referred to the oncologist. In January 2011, the patient reported again to her orthopedic surgeon due to pain she was experiencing in the surgical scar region, which radiated along the front of the thigh and lower leg. Control MRI performed in April 2011 revealed three locations of local recurrence: a mass $15 \times 43 \times 15 \mathrm{~mm}$ in size located between the two gluteal muscles - right gluteus medius 
and maximus; second mass with maximum dimensions $45 \times 120 \times 25 \mathrm{~mm}$ located under the subcutaneous fatty tissues from anterior iliac crest and caudal to greater trochanter of the right hip; and the third mass $18 \times 36 \times 15$ $\mathrm{mm}$ in size posterior in the middle third from the previously described mass (Figure 1 ). A smaller tumor mass sized $14 \times 22 \times 8 \mathrm{~mm}$ was described in ischioanal fossa, clearly differentiated from the surrounding tissue with intensive post-contrast enhancement, with three additional lesions, $20 \times 8 \mathrm{~mm}, 21 \times 8 \mathrm{~mm}$ and $9 \times 2 \mathrm{~mm}$ in size in the right internal obturator muscle. At this time, orthopedic surgeon recommended another control MRI in 6 months.

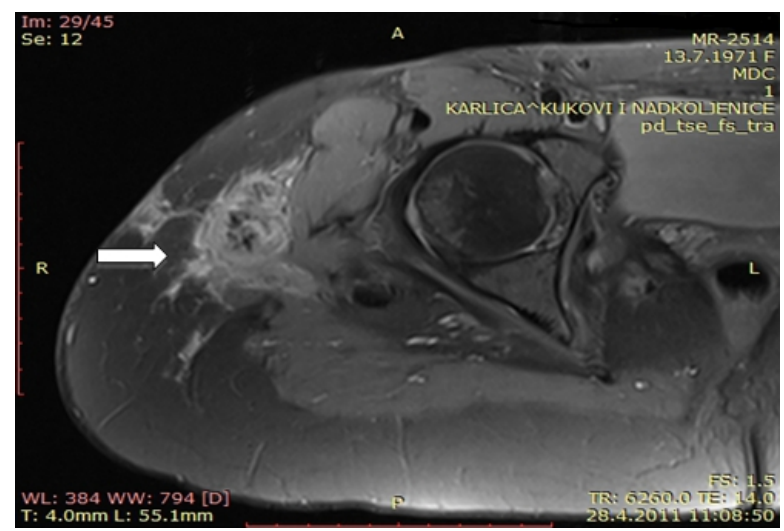

Figure 1: MRI scan performed in April 2011 showing three locations of disease recurrence.

Patient was referred to the oncologist for the first time in May 2012. Retrospective inspection of medical records and pathology report revealed that the patient had desmoid fibromatosis with low mitotic activity, with tumor cells positive for vimentin, SMA, myoglobin, and negative for desmin, MyoD1, with low proliferative Ki67 positivity but with tumor cells present at the resection margins. Patient was advised to repeat an MRI and to consult an orthopedist and a neurosurgeon. A control MRI performed in May 2012 revealed a progression in internal obturator muscle, with a possible infiltration of levator ani muscle (Figure 2). Orthopedic surgeon and a neurosurgeon were consulted and have advised against repeated surgical procedure due to the size of recurrent tumor mass. A multidisciplinary team held a case conference in August 2012 and advised against irradiation, due to high risk of radiotherapy-induced complications, considering the tumor size, invasion and proximity of the sciatic nerve. Therefore, the recommended treatment was systemic therapy with hormonal agent Tamoxifen $20 \mathrm{mg} /$ day and COX-II inhibitor Celebrex 200 mg. Three months later, a control MRI revealed signs of tumor necrosis, no further change in size and distribution of tumor lesions and no new lesions. Therefore, the same therapy was continued. Control MRI in February 2013 revealed no further disease progression and the therapy remained unchanged.

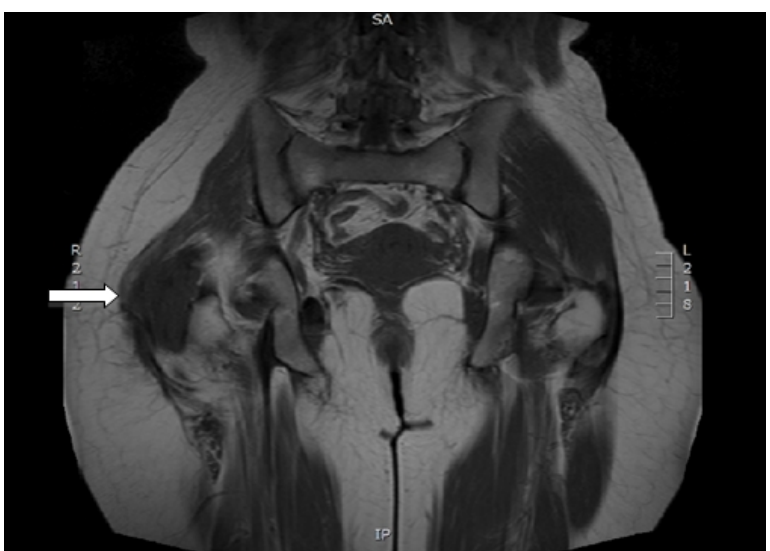

Figure 2: A control MRI performed in May 2012 describing a progressive recurrence of the disease.

An MRI performed in July 2013 displayed disease recurrence along the lesion of the right quadratus femoris and internal obturator muscles. Compared to the previous MRI, some lesions were larger and some were smaller in size, and there appeared to be a new lesion located in the right obturator internal muscle. Patient was prescribed interferon-alpha at the dose of 3.500.000 IU three times a week within 6 months, considering that the patient was not motivated for treatment with chemotherapy. Control MRI performed in March 2014 revealed that the largest lesion had shrunk in size while the smallest lesion had grown (Figure 3). At this time point, Imatinib was added to the list of essential medicines in Bosnia and Herzegovina, so, considering the results of the clinical trials ${ }^{6}$, the team recommended therapy with Imatinib. Patient initiated Imatinib therapy in April 2014. Control MRI in June 2014 revealed a decrease in size of the aforementioned tumor masses and lesions. Our patient continued therapy with Imatinib, without serious adverse events. The latest MRI scan performed in October 2015 showed complete regression of some lesions, no changes in size of other lesions, but with lower post-contrast enhancement and complete fibrosis of the largest lesion.

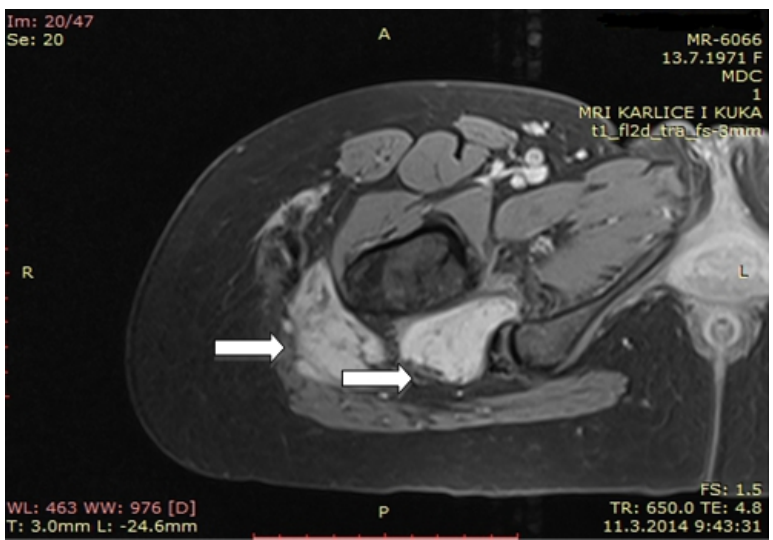

Figure 3: Control MRI performed in March 2014 showing regression of one lesion and progression of the other. 


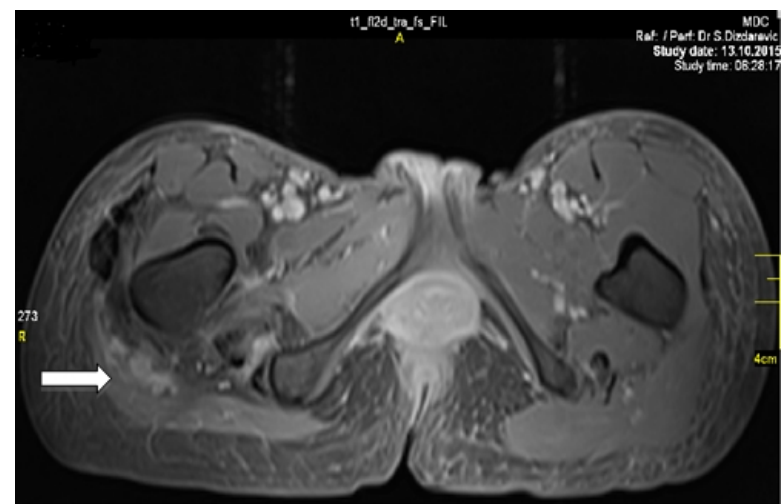

Figure 4: Control MRI performed in October 2015 showing complete regression of some lesions, other lesions without changes in size, but with lower post-contrast enhancement and complete fibrosis of the largest lesion.

\section{Discussion}

While DT is a benign tumor, it is not necessarily a benign disease. Within the general population, the incidence of DT is 2-4 cases per million people annually ${ }^{7}$. The incidence is higher in patients with familial adenomatous polyposis (FAP). DTs occur in $10-15 \%$ of these patients ${ }^{8}$. Its etiology is most likely multifactorial and includes genetic predisposition, endocrine factors and trauma. There are also sporadic forms without hereditary background. Most of the cases occur in the age between 15 and 60 years old, with the highest incidence between 25 and 35 years old ${ }^{3}$. DTs arise in connective tissue, fascia or aponeurosis of the muscle and tend to infiltrate adjacent muscle bundles, frequently entrapping them and causing their degeneration ${ }^{9}$. Typical clinical presentation includes a painless, slow-growing, deep-seated mass. DTs are commonly divided into abdominal and extra-abdominal tumors, based on their localization ${ }^{\mathbf{1 0}}{ }^{11}$. DTs lack the ability to metastasize, and therefore local control using surgery and radiation has traditionally been the mainstay of therapy for these tumors. Negative margin status (defined as surgical resection with microscopically negative margins) should be achieved, if possible, for a patient who is treated surgically ${ }^{2}$. Radiotherapy has been incorporated into the management of extra-abdominal DT, either as an adjuvant treatment after surgical resection in cases of positive surgical margins or as a primary treatment when surgical resection is not feasible or may result in significant loss of function ${ }^{4}$. In a review published by Melis et al. $^{12}$ it was suggested that surgical excision and radiotherapy in the treatment of recurrent lesions provide better results than surgery alone. The use of postoperative radiation, as described in a review of 22 series $^{13}$, increases the local control of the disease. In patients with positive margins, the local control increased from $4 \%$ to $75 \%$ with the addition of adjuvant irradiation. Positive effect of irradiation was also observed in patients with negative surgical margins. In case of disease relapse, there are several systemic treatment options, such as anti-hormonal therapy ${ }^{\mathbf{1 4}}$, non-steroidal anti-inflammatory drugs (NSAIDs) (e.g., cyclooxygenase [COX]-2 inhibitors $)^{15}$ classic chemotherapy regimens and tyrosine kinase inhibitor (Imatinib) ${ }^{5}$. Combinations of a non-steroidal anti-inflammatory drugs and Tamoxifen are commonly used as primary treatment of desmoids ${ }^{\mathbf{1 6}}$.

In a clinical trial performed by the French sarcoma group, there were positive initial results of treatment with Imatinib with non-progression rates of $90 \%$ and $80 \%$ at 3 and 6 months, respectively, but these decreased to $67 \%$ at 12 months. The median time to progression in this study was 25 months $^{6}$. These results were confirmed in the SARC (Sarcoma Alliance for Research through Collaboration) trial with initial progression-free survival of $94 \%$ and $88 \%$ at 2 - and 4-months follow-up appointments but these decreased significantly to $66 \%$ at 1 year ${ }^{17}$. Despite the limited potential of Imatinib alone, there is still an increasing interest for tyrosine kinase inhibitors in the treatment of extra-abdominal DTs.

Given the variability of the clinical course of DTs and the potential for complications that can arise as a result of therapy, the cases of all patients with DTs, who are about to undergo active treatment, should be discussed by an experienced multidisciplinary sarcoma team, and the treatment plan should take into consideration patient preferences. Even though the pathology report in our patient showed that tumor cells were present at the resection margins, due to the lack of multidisciplinary work, the patient did not receive radiotherapy. Our patient was presented for the first time at a multidisciplinary tumor board a year after disease relapse was diagnosed. Considering the risk of radiotherapy-induced complications ${ }^{\mathbf{1 8}}$ due to tumor size, multicentricity, invasion and proximity of sciatic nerve, the tumor board did not recommend radiotherapy. Therefore, the only treatment option was systemic therapy. There are no evidence-based or widely accepted guidelines for management of unresectable DT. Systemic therapy is increasingly being integrated into a multidisciplinary approach for selected patients with unresectable or intra-abdominal desmoids for which local therapy options may cause unacceptable morbidity. In the literature similar cases of recurrent DT have been described with an excellent response to Imatinib ${ }^{19}$.

\section{Conclusion}

After two lines of therapy, considering the results of other studies, our patient received treatment with tyrosine kinase inhibitor Imatinib, which led to a good partial response with acceptable side effects. It is important to emphasize that, due to financial challenges of the health care system in Bosnia, Imatinib is only financed in patients with gastrointestinal stromal tumor 
and chronic myeloid leukemia. Our patient was the first case of DT in Bosnia who received this treatment. We believe that the most important concept in the treatment of DT is to individualize each patient and to have a multidisciplinary approach involving the surgeon, radiation and medical oncologist, pathologist, radiologist, psychologist, occupational therapist, physiotherapist and most importantly to always take into account the patient's opinion. With this case report we hope to contribute to better understanding of this rare disease and that it might be a useful asset in choosing the treatment of these patients.

\section{Conflict of interest}

The authors declare that they have no conflicts of interest. The authors alone are responsible for the content and writing of the paper.

\section{References}

1. Shields CJ, Winter DC, Kirwan WO, et al. Desmoid tumours. Eur J Surg Oncol. 2001;27(8):701-6.

2. Gronchi A, Casali PG, Mariani L, et al. Quality of surgery and outcome in extra-abdominal aggressive fibromatosis: a series of patients surgically treated at a single institution. J Clin Oncol. 2003;21(7):1390-7.

3. Kasper B, Strobel P, Hohenberger P. Desmoid tumors: clinical features and treatment options for advanced disease. Oncologist. 2011;16(5):682-93.

4. Micke 0, Seegenschmiedt MH. Radiation therapy for aggressive fibromatosis (desmoid tumors): results of a national Patterns of Care Study. Int J Radiat Oncol Biol Phys. 2005;61(3):882-91.

5. Mace J, Sybil Biermann J, Sondak V, et al. Response of extraabdominal desmoid tumors to therapy with imatinib mesylate. Cancer. 2002;95(11):2373-9.

6. Penel N, Le Cesne A, Bui BN, et al. Imatinib for progressive and recurrent aggressive fibromatosis (desmoid tumors): an FNCLCC/French Sarcoma Group phase II trial with a long-term follow-up. Ann Oncol. 2011;22(2):452-7.

7. Reitamo JJ, Hayry P, Nykyri E. The desmoid tumor. I. Incidence, sex-, age- and anatomical distribution in the Finnish population. Am J Clin Pathol. 1982;77(6):665-73.
8. Brueckl WM, Ballhausen WG, Fortsch T, et al. Genetic testing for germline mutations of the APC gene in patients with apparently sporadic desmoid tumors but a family history of colorectal carcinoma. Dis Colon Rectum. 2005;48(6):1275-81.

9. Wu C, Amini-Nik S, Nadesan P, et al. Aggressive fibromatosis (desmoid tumor) is derived from mesenchymal progenitor cells. Cancer Res. 2010;70(19):7690-8.

10. De Bree E, Keus R, Melissas J, et al. Desmoid tumors: need for an individualized approach. Expert Rev_Anticancer Ther. 2009;9(4):525-35.

11. Ferenc T, Sygut J, Kopczynski J, et al. Aggressive fibromatosis (desmoid tumors): definition, occurrence, pathology, diagnostic problems, clinical behavior, genetic background. Pol J Pathol. 2006;57(1):5-15.

12. Melis M, Zager JS, Sondak VK. Multimodality management of desmoid tumors: how important is a negative surgical margin? J Surg Oncol. 2008;98(8):594-602.

13. Nuyttens JJ, Rust PF, Thomas CR Jr., et al. Surgery versus radiation therapy for patients with aggressive fibromatosis or desmoid tumors: A comparative review of 22 articles. Cancer. 2000;88(7):1517-23.

14. Hansmann A, Adolph C, Vogel T, et al. High-dose tamoxifen and sulindac as first-line treatment for desmoid tumors. Cancer. 2004;100(3):612-20.

15. Nishida Y, Tsukushi S, Shido Y, et al. Successful treatment with meloxicam, a cyclooxygenase-2 inhibitor, of patients with extra-abdominal desmoid tumors: a pilot study. J Clin Oncol. 2010;28(6):e107-9.

16. Janinis J, Patriki M, Vini L, et al. The pharmacological treatment of aggressive fibromatosis: a systematic review. Ann Oncol. 2003;14(2):181-90.

17. Chugh R, Wathen JK, Patel SR, et al. Efficacy of imatinib in aggressive fibromatosis: Results of a phase II multicenter Sarcoma Alliance for Research through Collaboration (SARC) trial. Clin Cancer Res. 2010;16(19):4884-91.

18. Rutenberg MS, Indelicato DJ, Knapik JA, et al. External-beam radiotherapy for pediatric and young adult desmoid tumors. Pediatr Blood Cancer. 2011;57(3):435-42.

19. Molloy AP, Hutchinson B, O'Toole GC. Extra-abdominal desmoid tumours: a review of the literature. Sarcoma. 2012;2012:578052. 